\title{
Validation of asthma recording in electronic health records: a systematic review
}

This article was published in the following Dove Press journal:

Clinical Epidemiology

\section{Francis Nissen' \\ Jennifer K Quint ${ }^{2}$ \\ Samantha Wilkinson' \\ Hana Mullerova ${ }^{3}$ \\ Liam Smeeth' \\ Ian J Douglas'}

'Department of Non-Communicable Disease Epidemiology, London School of Hygiene and Tropical Medicine, London, UK; ${ }^{2}$ National Heart and Lung Institute, Imperial College, London, UK; ${ }^{3}$ RWD \& Epidemiology, GSK R\&D, Uxbridge, UK
Correspondence: Francis Nissen Department of Non-Communicable Disease Epidemiology, London School of Hygiene and Tropical Medicine, Keppel Street, London, WCIE 7HT, UK Email francis.nissen@Ishtm.ac.uk
Objective: To describe the methods used to validate asthma diagnoses in electronic health records and summarize the results of the validation studies.

Background: Electronic health records are increasingly being used for research on asthma to inform health services and health policy. Validation of the recording of asthma diagnoses in electronic health records is essential to use these databases for credible epidemiological asthma research.

Methods: We searched EMBASE and MEDLINE databases for studies that validated asthma diagnoses detected in electronic health records up to October 2016. Two reviewers independently assessed the full text against the predetermined inclusion criteria. Key data including author, year, data source, case definitions, reference standard, and validation statistics (including sensitivity, specificity, positive predictive value [PPV], and negative predictive value [NPV]) were summarized in two tables.

Results: Thirteen studies met the inclusion criteria. Most studies demonstrated a high validity using at least one case definition (PPV $>80 \%$ ). Ten studies used a manual validation as the reference standard; each had at least one case definition with a PPV of at least $63 \%$, up to $100 \%$. We also found two studies using a second independent database to validate asthma diagnoses. The PPVs of the best performing case definitions ranged from $46 \%$ to $58 \%$. We found one study which used a questionnaire as the reference standard to validate a database case definition; the PPV of the case definition algorithm in this study was $89 \%$.

Conclusion: Attaining high PPVs ( $>80 \%)$ is possible using each of the discussed validation methods. Identifying asthma cases in electronic health records is possible with high sensitivity, specificity or PPV, by combining multiple data sources, or by focusing on specific test measures. Studies testing a range of case definitions show wide variation in the validity of each definition, suggesting this may be important for obtaining asthma definitions with optimal validity.

Keywords: sensitivity, specificity, PPV, NPV, database, validity, epidemiology

\section{Background}

Asthma is one of the most common chronic diseases, and its core symptoms are cough, wheeze, breathlessness, and chest tightness. ${ }^{1}$ There is no cure, but with the right treatment, symptoms ranging from mild attacks to severe and life-threatening exacerbations ${ }^{2}$ can be managed. ${ }^{1}$ Despite this, a sizeable percentage of asthma patients are poorly controlled. ${ }^{3,4}$

Electronic health records (EHRs) have been widely adopted, which allows for the construction of large population-based patient databases. The availability of these routinely generated longitudinal records for research has greatly increased over the 
last decades. ${ }^{5}$ However, the accuracy of diagnoses recorded in these large databases may be low, which would introduce bias into studies using the data. Unless the data are validated for research, the quality of studies generated from EHRs may be debatable. ${ }^{6-9}$ Furthermore, the validity of different disease definitions is not always the same in a given dataset. Some diseases (such as asthma) might be coded using less specific symptoms, whereas the validity of diagnoses with very specific symptoms (such as tension pneumothorax) is likely to be better.

EHRs predominantly store information about diagnoses as clinical codes. A single code, or a case definition consisting of multiple codes (with or without additional information such as tests or prescribing) can be used to retrieve records from EHRs, and additional restrictions can be applied such as age or exclusion of other diseases. ${ }^{9,10}$ Validity of coding is generally assessed by comparing a code (or algorithm) to 1) the diagnosis as verified by the treating physician either by manual review of the chart notes or in clinic, 2) a reference standard such as another linked dataset, or 3) a patient questionnaire. ${ }^{10}$ A previous systematic review by Sharifi et al reviewed validation methods to capture acute bronchospasm in administrative or claims data; ${ }^{11}$ this review identified two validation studies of bronchospasm codes. ${ }^{12,13}$ However, the study was limited to administrative and claims databases, from the United States and Canada. Al Sallakh et al explored approaches to defining asthma or assessing asthma outcomes using EHR-derived data in the recent literature (calendar years 2014 and 2015) and examined the clarity of reporting. ${ }^{14}$ This systematic review focuses on how asthma was defined and does not include an overview of test measures or validation statistics.

There is currently no consensus on approaches to defining asthma or assessing asthma outcomes using EHR-derived data. We explored these approaches in the recent literature and examined the clarity of reporting.

\section{Research objective}

The primary objectives of this systematic review are to provide an overview of the methods used in the literature for validating asthma diagnosis in EHRs, and the corresponding estimates of the validation test measures.

\section{Methods}

The methods are described in detail in the study protocol. ${ }^{15}$ We searched MEDLINE and EMBASE up to October 2016 for relevant articles. Our search strategy was composed of the following sets of terms: 1) electronic health records or databases AND 2) validity or validation or case definition or algorithm or sensitivity or specificity or positive predictive value or negative predictive value AND 3) the medical subject heading terms for asthma. Reference lists of articles of interest were reviewed to add potential additional studies in which a validation of asthma diagnosis was done. The PRISMA flow diagram can be found in Figure 1 and the search strategy can be found in the supplementary material. We considered any type of observational study design that used EHR to validate the recording of a diagnosis of asthma. In addition, we required a clear case definition to define asthma from EHR, including a description of the validation of said case definition through at least one test measure (sensitivity, specificity, positive predictive value [PPV] or negative predictive value [NPV]). Two investigators (FN and SW) separately assessed the abstracts and full text of each potential study against our inclusion criteria; disagreements were resolved through a third investigator or by discussion to reach consensus. The first author extracted all relevant data regarding methodologic elements of included studies; author, year of publication, country, time period, date, data source, population, case characteristics, clinical events, algorithms, reference standard, and validation statistics. Bias was assessed using QUADAS-2 tailored to this specific review. ${ }^{16}$

The questions of interest for this systematic review are: 1) which EHR databases were used to obtain information on the diagnosis of asthma? 2) Which case definitions, algorithms or codes were used to define an asthma diagnosis? 3) How were the diagnostic criteria applied to the data sources and which other approaches have been used to validate a case definition algorithm? and 4) What are the estimates for the PPV, NPV, specificity, and sensitivity for a diagnosis of asthma in an EHR?

\section{Inclusion criteria}

Any type of observational study design which validated the recording of an asthma diagnosis in EHR was considered. Articles were only considered if published in English and published before October 2016 without any specific start date. Within the databases, we considered asthma diagnoses based on both structured data (such as laboratory results and prescriptions) and unstructured data (such as spirometry results). We required the validation case definitions to be compared to an external reference standard, such as a manual review, questionnaires (completed by the patient or their physician) or an independent second database. We included case definitions formed of single codes, those requiring multiple case characteristics, and case definitions generated by natural language processing (NLP) and/or machine-learning. 


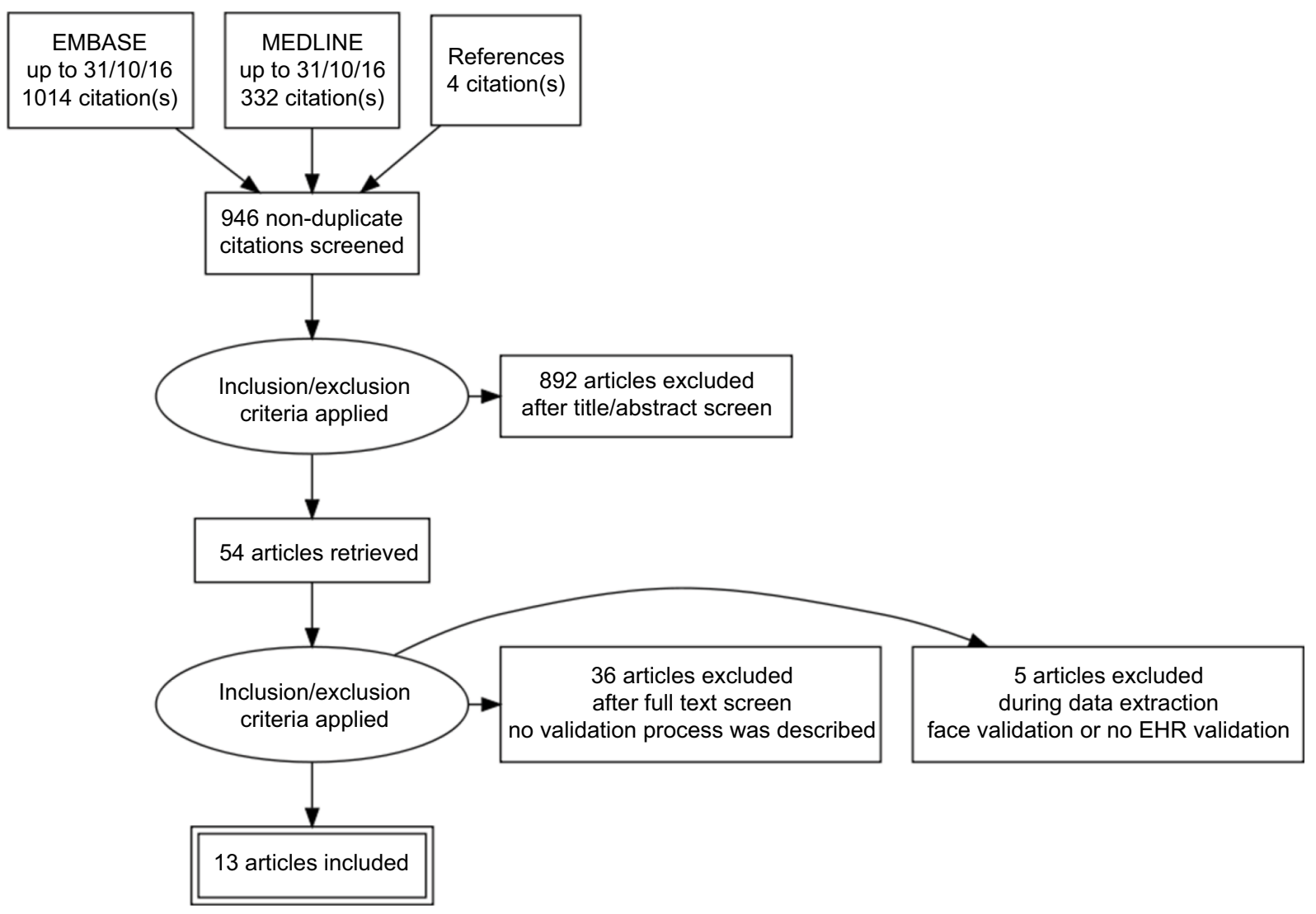

Figure I Study screening process: PRISMA flow diagram.

Note: Reproduced from Moher D, Liberati A, Tetzlaff J, Altman DG, PRISMA Group. Preferred reporting items for systematic Reviews and meta-analyses: the PRISMA statement. BMJ. 2009;339:b2535. ${ }^{37}$

Abbreviation: EHR, electronic health record.

\section{Exclusion criteria}

EHRs are a digital reflection of the key facts a health care provider needs to record in order to facilitate ongoing and potentially complex clinical care. By contrast, the main purpose of administrative claims data is administration of reimbursements to health care providers for their services. This systematic review included only studies from EHRs, as the quality measures between the two types of data can be markedly different; studies using administrative claims data were excluded. Studies involving pharmacovigilance databases (signal detection or spontaneous reporting), studies without validation of asthma recording, and conference abstracts were excluded..$^{17,18}$

\section{Data synthesis}

Studies and study data were managed using EndNote and Microsoft Excel, respectively.

The methods for validation of asthma recording in the included studies were outlined in a narrative synthesis. In addition, Table 1 summarizes the methods and Table 2 describes the results, consisting of the recorded PPV, NPV, sensitivity, and specificity of the included studies.

\section{Dissemination and ethics}

This study is a synthesis of previously published studies, so no ethical approval is required. The protocol was registered in the PROSPERO database with registration number CRD42016041798, and the protocol has been published. ${ }^{15}$ Results from this systematic review can be used to study outcome research on asthma and can be used to identify case definitions for asthma.

\section{Results}

In total, 1,346 titles were found in the EMBASE and MEDLINE databases, of which 946 were non-duplicates. Of those, 54 articles were reviewed in full text and we found 13 articles that contained a validation process of asthma diagnosis that met all eligibility criteria. Characteristics of the 13 included studies ordered by year of publication are summarized in Table 1, and the study results are displayed in Table 2 . The asthma prevalence necessary for the interpretation of PPVs and NPVs is presented in Table 1, where available.

The reference standard used to validate the asthma diagnosis in the EHRs differed between the studies: ten studies used manual validation by a clinician, two studies compared 


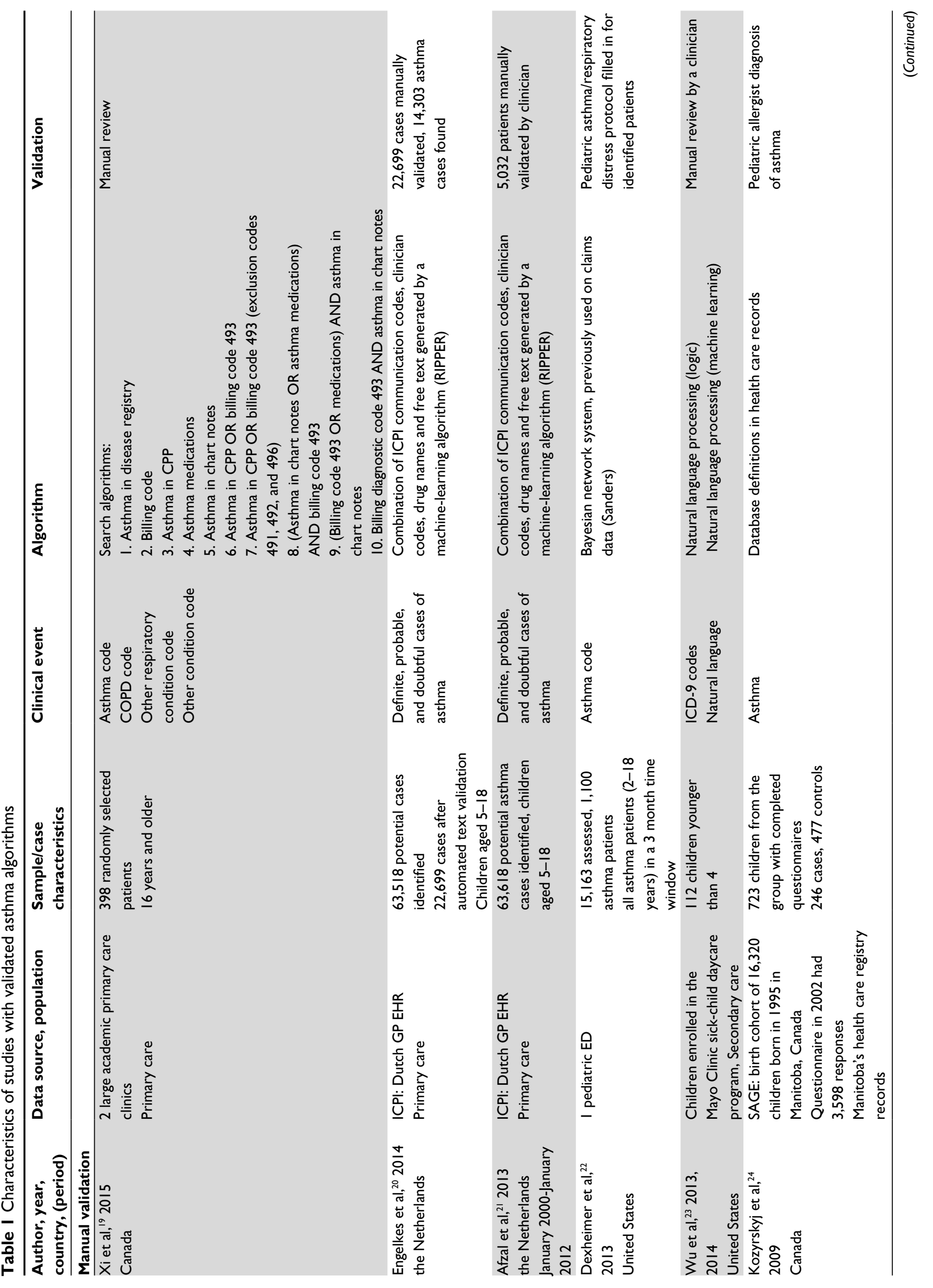




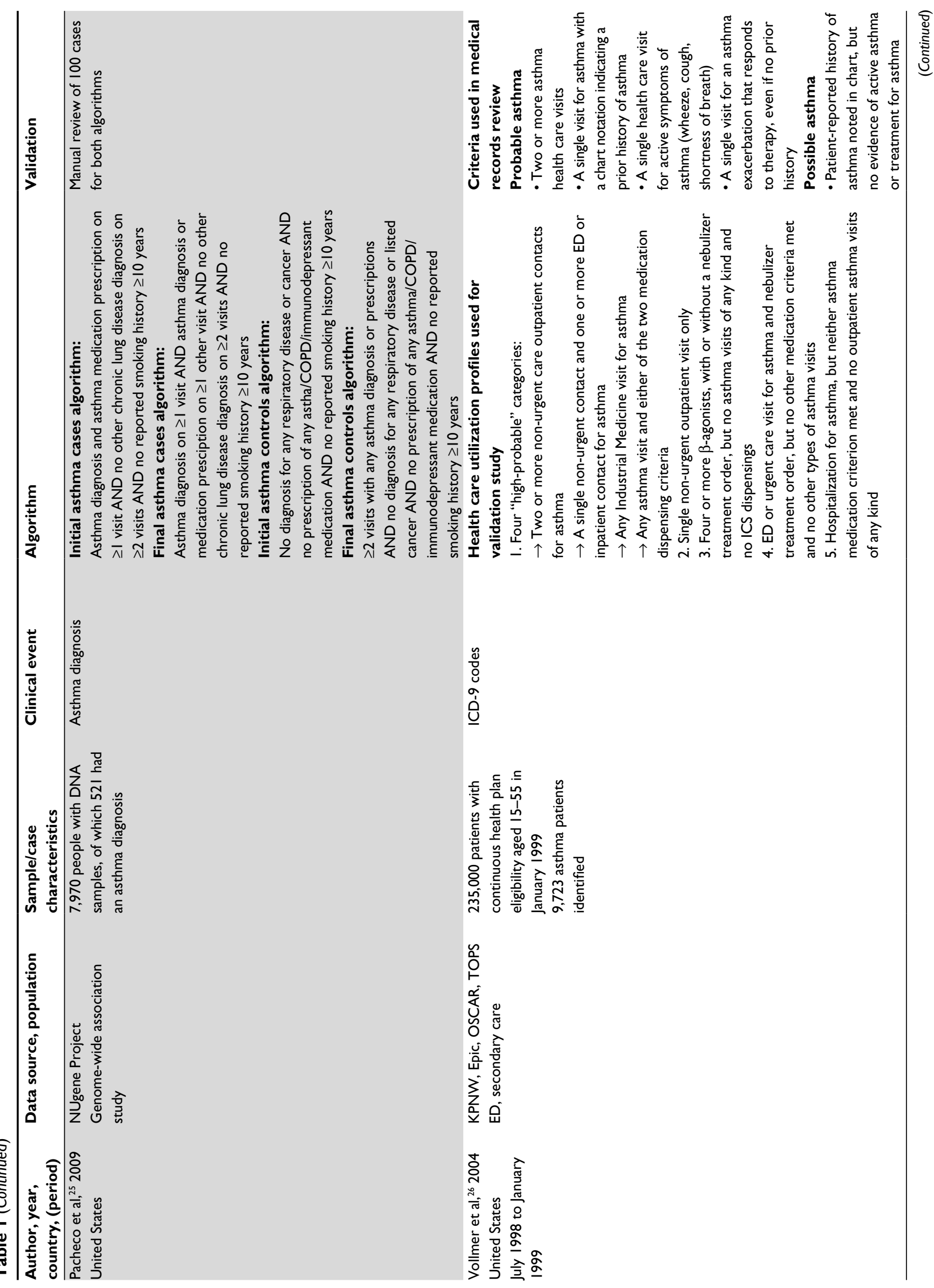




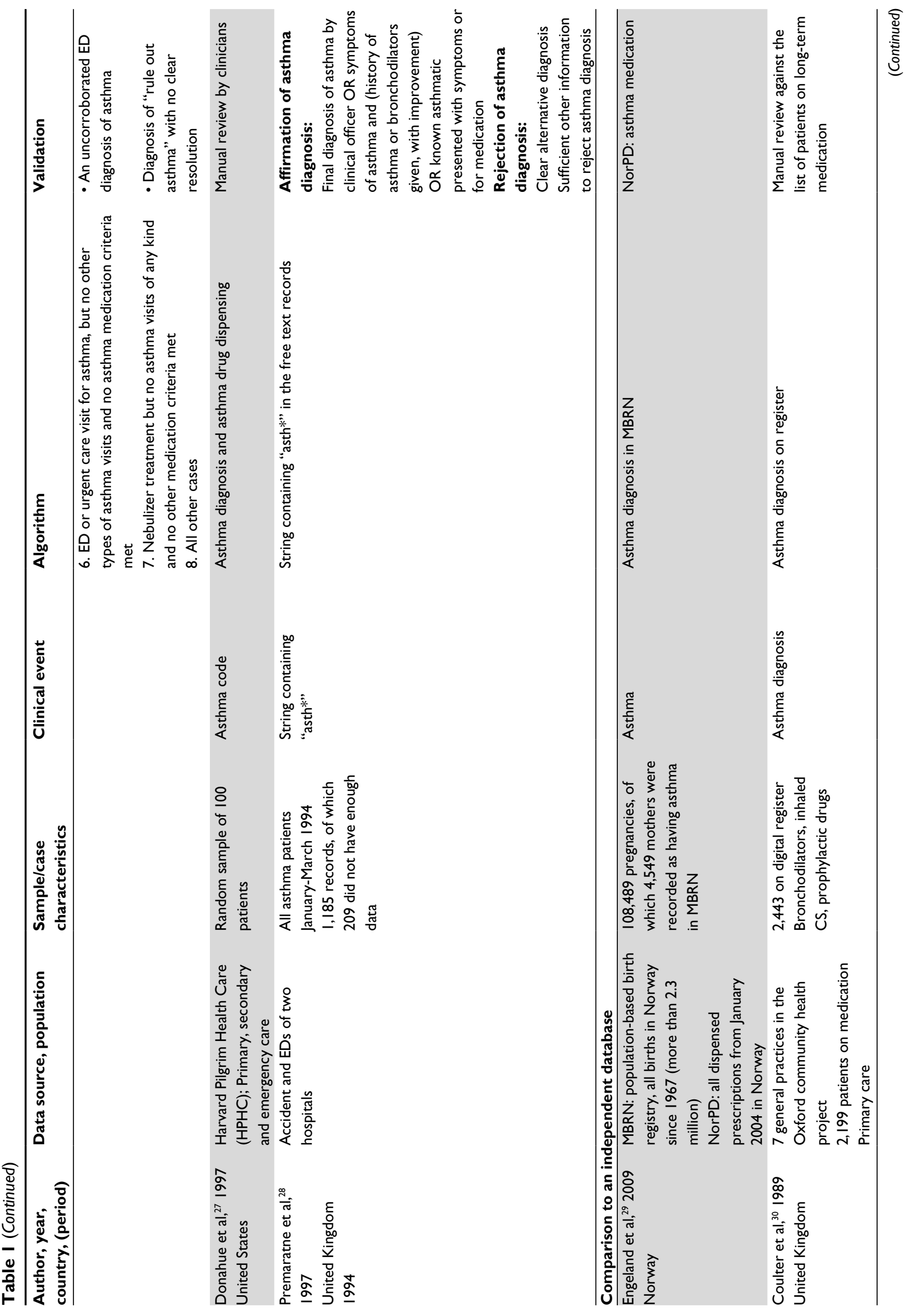




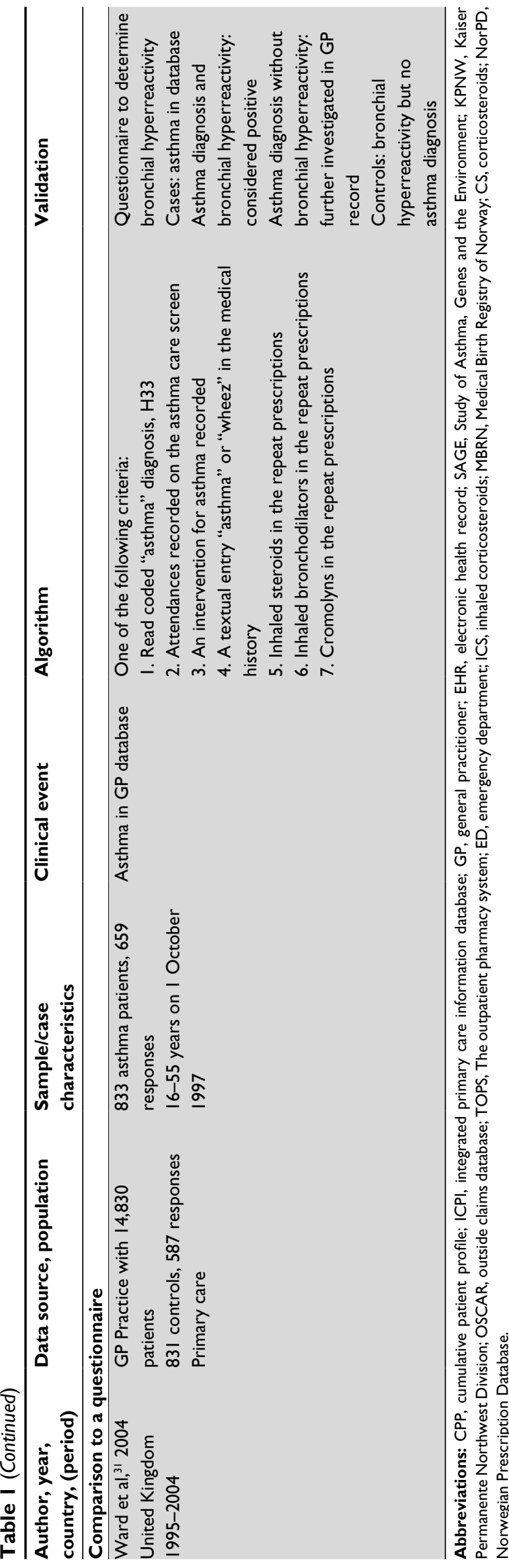

the studied records to independent linked databases and one study used patient questionnaires. The test measures also differ between the different papers, encompassing sensitivity, specificity, PPV, and NPV. We focus on 13 studies in this review, ordered by reference standard used and by date of publication. Bias assessment results using QUADAS-2 are presented in Table 3.

\section{Manual validation}

We found ten studies that used a manual validation as the reference standard. All studies had at least one case definition algorithm with a PPV of at least $63 \%$. Where other measurements could be calculated, the studies had at least one case definition with a sensitivity of at least $85 \%$, specificity of at least $92 \%$, and NPV of at least $94 \%$. Within this group, four studies used case definition algorithms generated by machine learning. Five studies included only children, while two studies included only persons older than 16 years.

$\mathrm{Xi}$ et al tested a variety of EHR search algorithms based on two large academic primary care clinics in Hamilton, Canada. ${ }^{19}$ The reference standard consisted of a physician chart review-based diagnosis. The eight case definitions are presented in Table 1, and their PPVs in Table 2. The algorithm searching for patients who had asthma in their patient profile or had an asthma billing code was the most accurate with a sensitivity of $90 \%$ (95\% CI [ $87 \%$ to $93 \%])$ and a specificity of $84 \%$ (95\% CI [ $80 \%$ to $88 \%])$.

Engelkes et al undertook a study to determine the validity of case definitions generated by machine learning to define asthma cases, based on a previous study be Afzal et al..$^{20,21}$ Originating from a large Dutch general practitioner (GP) database, the authors manually reviewed 22,699 potential asthma cases. Among those, 14,303 asthma cases were found, which resulted in a PPV of $63 \%$.

The study by Afzal et al uses the same dataset and machine-learning algorithm for definite and potential asthma cases as the study by Engelkes et al. ${ }^{20,21}$ Clinicians manually validated 5,032 potential asthma cases identified by a broad search algorithm out of 63,618 patients. This training set was used for the machine-learning algorithm. The test measures are measuring the validity of the machine-learning algorithm within the smaller population, not of the broad search algorithm. The PPV, sensitivity, and specificity for three case definition algorithms (definite cases; definite and probable cases; definite, probable, and doubtful cases) were calculated. The PPVs range from $57 \%$ for all definite, probable, and doubtful asthma cases to $82 \%$ for only the definite asthma cases. 
Table 2 Characteristics of studies with validated asthma algorithms

\begin{tabular}{|c|c|c|c|c|c|c|}
\hline $\begin{array}{l}\text { Author, year, } \\
\text { country, prevalence }\end{array}$ & Algorithm & $\begin{array}{l}\text { Sensitivity, } \\
95 \% \mathrm{Cl}\end{array}$ & $\begin{array}{l}\text { Specificity, } \\
95 \% \mathrm{Cl}\end{array}$ & $\begin{array}{l}\text { PPV, } \\
95 \% \mathrm{Cl}\end{array}$ & $\begin{array}{l}\text { NPV, } \\
95 \% \mathrm{Cl}\end{array}$ & Prevalence \\
\hline \multicolumn{7}{|l|}{ Manual validation } \\
\hline Xi et al, ${ }^{19} 2015$ & I. Asthma in disease registry & $7 \%(5-10)$ & $99 \%(97-100)$ & $67 \%(38-87)$ & $73 \%(72-74)$ & $8.1 \%$ \\
\hline \multirow[t]{9}{*}{ Canada } & 2. Billing code & $77 \%(75-83)$ & $89.2 \%(86-92)$ & $74 \%(67-80)$ & $91 \%(88-94)$ & \\
\hline & 3. Asthma in CPP & $63 \%(59-68)$ & $92 \%(90-95)$ & $76 \%(68-83)$ & $87 \%(83-89)$ & \\
\hline & 4. Asthma medications & $79 \%(75-83)$ & $64 \%(59-68)$ & $46 \%(4 I-50)$ & $88 \%(84-92)$ & \\
\hline & 5. Asthma in chart notes & $85 \%(81-88)$ & $76 \%(72-80)$ & $58 \%(52-63)$ & $93 \%(89-95)$ & \\
\hline & 6. Asthma in CPP OR billing code 493 & $90 \%(87-93)$ & $84 \%(80-88)$ & $69 \%(63-74)$ & $96 \%(93-97)$ & \\
\hline & $\begin{array}{l}\text { 7. Asthma in CPP OR billing code } 493 \\
\text { (exclusion codes } 491,492 \text {, and } 496 \text { ) }\end{array}$ & $87 \%(83-90)$ & $85 \%(82-89)$ & $70 \%(63-76)$ & $94 \%(9 \mid-96)$ & \\
\hline & $\begin{array}{l}\text { 8. (Asthma in chart notes OR asthma } \\
\text { medications) AND billing code } 493\end{array}$ & $78 \%(74-82)$ & $92 \%(89-95)$ & $79 \%(72-85)$ & $91 \%(88-94)$ & \\
\hline & $\begin{array}{l}\text { 9. (Billing code } 493 \text { OR medications) } \\
\text { AND asthma in chart note }\end{array}$ & $84 \%(80-88)$ & $84 \%(80-88)$ & $67 \%(6 I-73)$ & $93 \%(90-95)$ & \\
\hline & $\begin{array}{l}\text { 10. Billing diagnostic code } 493 \text { AND } \\
\text { asthma in chart notes }\end{array}$ & $74 \%(70-78)$ & $93 \%(91-96)$ & $81 \%(73-87)$ & $90 \%(87-93)$ & \\
\hline Engelkes et al,,$^{20} 2014$ & Definite, probable and doubtful cases & & & $63 \%$ & & \\
\hline \multicolumn{7}{|l|}{ Netherlands } \\
\hline Afzal et al, ${ }^{21} 2013$ & Definite asthma & $98 \%$ & $95 \%$ & $66 \%$ & & $6 \%$ \\
\hline \multirow[t]{2}{*}{ Netherlands } & Definite + probable & $96 \%$ & $90 \%$ & $82 \%$ & & $29 \%$ \\
\hline & Definite, probable and doubtful cases & $95 \%$ & $67 \%$ & $57 \%$ & & $32 \%$ \\
\hline $\begin{array}{l}\text { Dexheimer et al, }{ }^{22} \\
2013\end{array}$ & $\begin{array}{l}\text { Algorithm constructed using a Bayesian } \\
\text { network system }\end{array}$ & & & $64 \%$ & & $7-10 \%$ \\
\hline \multicolumn{7}{|l|}{ United States } \\
\hline Wu et al, ${ }^{23} 2013,2014$ & ICD-9 codes & 31 & 93 & 57 & 82 & $4-17 \%$ \\
\hline \multirow[t]{2}{*}{ United States } & Natural language processing: logic & 81 & 95 & 84 & 94 & \\
\hline & $\begin{array}{l}\text { Natural language processing: machine } \\
\text { learning }\end{array}$ & 85 & 97 & 88 & 95 & \\
\hline \multirow[t]{6}{*}{$\begin{array}{l}\text { Kozyrskyj et al, }{ }^{24} 2009 \\
\text { Canada }\end{array}$} & $\begin{array}{l}\text { At least one asthma hospitalization, or } \\
\text { two physician visits, or four prescription } \\
\text { medications }\end{array}$ & $47 \%(35-60)$ & $92 \%(78-98)$ & $91 \%(76-98)$ & & $11 \%$ \\
\hline & $\begin{array}{l}\text { At least one asthma hospitalization, or } \\
\text { two physician visits, or two prescription } \\
\text { medications }\end{array}$ & $67 \%(54-78)$ & $92 \%(78-98)$ & $94 \%(82-99)$ & & \\
\hline & $\begin{array}{l}\text { At least one asthma hospitalization, or } \\
\text { one physician visit, or two prescription } \\
\text { medications }\end{array}$ & $77 \%(65-87)$ & $92 \%(78-98)$ & $94 \%(85-99)$ & & \\
\hline & $\begin{array}{l}\text { At least one asthma hospitalization, } \\
\text { or one physician visit, or two } \\
\text { bronchodilators, or one controller } \\
\text { medication }\end{array}$ & $80 \%(69-89)$ & $89 \%(74-97)$ & $93 \%(83-98)$ & & \\
\hline & $\begin{array}{l}\text { At least one asthma hospitalization, } \\
\text { or one physician visit, or two } \\
\text { bronchodilators, or one bronchodilator } \\
\text { and ketotifen or an oral steroid, or one } \\
\text { controller medication }\end{array}$ & $80 \%(69-89)$ & $89 \%(74-97)$ & $93 \%(83-98)$ & & \\
\hline & $\begin{array}{l}\text { At least one asthma hospitalization, } \\
\text { or one physician visit, or one } \\
\text { bronchodilator, or one controller } \\
\text { medication }\end{array}$ & $82 \%(70-90)$ & $83 \%(67-94)$ & $90 \%(79-96)$ & & \\
\hline
\end{tabular}


Table 2 (Continued)

\begin{tabular}{|c|c|c|c|c|c|c|}
\hline $\begin{array}{l}\text { Author, year, } \\
\text { country, prevalence }\end{array}$ & Algorithm & $\begin{array}{l}\text { Sensitivity, } \\
95 \% \mathrm{Cl}\end{array}$ & $\begin{array}{l}\text { Specificity, } \\
95 \% \mathrm{Cl}\end{array}$ & $\begin{array}{l}\text { PPV, } \\
95 \% \mathrm{CI}\end{array}$ & $\begin{array}{l}\text { NPV, } \\
95 \% \mathrm{CI}\end{array}$ & Prevalence \\
\hline Pacheco et al, 2009 & Initial algorithm & $70 \%(60-78)$ & $100 \%$ & $100 \%(90-100)$ & $77 \%(65-86)$ & $7.2 \%$ \\
\hline United States & Final algorithm & $95 \%(84-99)$ & $96 \%(87-99)$ & $95 \%(84-99)$ & $96 \%(87-99)$ & \\
\hline Vollmer et al, ${ }^{26} 2004$ & Algorithm I: population of 4460 & & & $95 \%$ & & $4.1 \%$ \\
\hline \multirow[t]{7}{*}{ United States } & Algorithm 2: population of 2334 & & & $90 \%$ & & \\
\hline & Algorithm 3: population of 545 & & & $70 \%$ & & \\
\hline & Algorithm 4: population of 25 & & & $100 \%$ & & \\
\hline & Algorithm 5: population of II & & & $50 \%$ & & \\
\hline & Algorithm 6: population of 721 & & & $80 \%$ & & \\
\hline & Algorithm 7: population of 99 & & & $27 \%$ & & \\
\hline & Algorithm 8: population of 1528 & & & $80 \%$ & & \\
\hline \multicolumn{6}{|l|}{ United States } & $3 \%$ \\
\hline $\begin{array}{l}\text { Premaratne et al, }{ }^{28} \\
1997\end{array}$ & $\begin{array}{l}\text { String containing asth* in free text } \\
\text { records }\end{array}$ & $80 \%(75-86)$ & $96 \%(96-99)$ & $91 \%(87-94)$ & $94 \%(93-95)$ & $20.6 \%$ \\
\hline \multicolumn{7}{|l|}{ United Kingdom } \\
\hline \multicolumn{7}{|c|}{ Comparison to an independent database } \\
\hline $\begin{array}{l}\text { Engeland et al, }{ }^{29} 2009 \\
\text { Norway }\end{array}$ & Asthma in MBRN and NorPD & $51 \%(49-52)$ & $98 \%(98-98)$ & $46 \%(45-48)$ & & $4.20 \%$ \\
\hline $\begin{array}{l}\text { Coulter et al, }{ }^{30} 1989 \\
\text { United Kingdom }\end{array}$ & $\begin{array}{l}\text { Percentage of people on long term } \\
\text { medication and recorded on the register }\end{array}$ & & & $58 \%$ & & \\
\hline \multicolumn{7}{|c|}{ Comparison to a questionnaire } \\
\hline Ward et al, ${ }^{3 \prime} 2004$ & Total of all reviewed patients & & & $89 \%$ & & $5.60 \%$ \\
\hline \multirow[t]{2}{*}{ United Kingdom } & Cases without bronchial hyperreactivity & & & $73 \%$ & & \\
\hline & Controls with bronchial hyperreactivity & & & $78 \%$ & & \\
\hline
\end{tabular}

Abbreviations: PPV, positive predictive value; NPV, negative predictive value; NLP, natural language processing; ML, machine learning; MBRN, Medical Birth Registry of Norway; NorPD, Norwegian Prescription Database.

Dexheimer et al evaluated a computerized asthma detection system in an urban, tertiary care pediatric emergency department in a 3-month prospective, randomized controlled trial in 2009.22 A Bayesian network system screened all emergency department patients for acute asthma. The system identified 1,100 patients with asthma exacerbations, of which 704 were confirmed by a pediatric emergency care physician within 3 days of the visit. The PPV for the Bayesian network system was $65 \%$.

$\mathrm{Wu}$ et al evaluated the accuracy of a computational approach to asthma ascertainment. The authors developed an NLP system for extracting predetermined asthma from free text in EHRs. ${ }^{23}$ Manual chart review by a clinician was the reference standard. The patient group consisted of 112 children younger than 4 years. The NLP-generated case definition algorithms had a sensitivity of $85 \%$, specificity of $97 \%$, PPV of $88 \%$, and an NPV of $95 \%$. For compari- son, the test measures of the ICD-9 asthma codes were calculated (sensitivity $31 \%$, specificity $93 \%$, PPV 57\%, NPV 82\%).

Kozyrskyj et al described the Study of Asthma, Genes and the Environment (SAGE). The study captures the longitudinal health care records of 16,320 children born in 1995 in Manitoba (Canada) and contains detailed information on early-life exposures in relationship to the development of asthma. ${ }^{24}$ Within the birth cohort, a nested case-control study with 723 children was partly created to confirm asthma status in children and these data were used to validate health care database measures of asthma. These 723 children were chosen by random sampling from the birth cohort; the parents of 288 children with and 435 without a parental report of asthma in the last 12 months agreed to participate. The reference standard for the validation consisted of pediatric allergist-diagnosed asthma, methacholine challenge tests, and 
Table 3 Quality assessment using QUADAS-2

\begin{tabular}{|c|c|c|c|c|}
\hline \multirow[t]{2}{*}{ Study } & \multicolumn{4}{|c|}{ Risk of bias } \\
\hline & $\begin{array}{l}\text { Patient } \\
\text { selection }\end{array}$ & $\begin{array}{l}\text { Index } \\
\text { test }\end{array}$ & $\begin{array}{l}\text { Reference } \\
\text { standard }\end{array}$ & $\begin{array}{l}\text { Flow } \\
\text { and } \\
\text { timing }\end{array}$ \\
\hline Xi et al, ${ }^{19} 2015$ & (;) & $?$ & (:) & $?$ \\
\hline Engelkes et $\mathrm{al}^{20} 2014$ & (:) & (:) & $:$ & (:) \\
\hline Afzal et al, ${ }^{21} 2013$ & 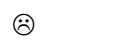 & (:) & (:) & ;) \\
\hline Dexheimer et al,,22 2013 & (:) & (:) & $\theta$ & (:) \\
\hline Wu et al, ${ }^{23} 2013,2014$ & : & (:) & $?$ & ;) \\
\hline Kozyrskyj et al, ${ }^{24} 2009$ & 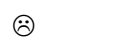 & 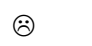 & (:) & (:) \\
\hline Pacheco et al, ${ }^{25} 2009$ & 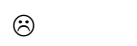 & (:) & (:) & (:) \\
\hline Vollmer et al, ${ }^{26} 2004$ & 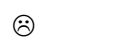 & (:) & (:) & (:) \\
\hline Donahue et al, ${ }^{27} 1997$ & (:) & 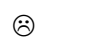 & $\theta$ & (:) \\
\hline Premaratne et al, ${ }^{28} 1997$ & (:) & 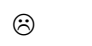 & (:) & (:) \\
\hline Engeland et al, ${ }^{29} 2009$ & : & $:$ & $\theta$ & 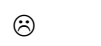 \\
\hline Coulter et al, ${ }^{30} 1989$ & 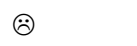 & 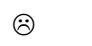 & $\theta$ & $?$ \\
\hline Ward et al, 2004 & 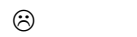 & : & (:) & : \\
\hline
\end{tabular}

Note: Happy face: low risk; sad face: high risk; question mark: unclear risk.

skin tests. The PPV of asthma definitions varied from $90 \%$ to $94 \%$, the sensitivity from $47 \%$ to $82 \%$, and the specificity from $83 \%$ to $92 \%$.

Pacheco et al constructed case definitions to identify asthmatic patients as cases, and healthy patients as controls using data from electronic medical records in the United States. This was done to identify asthma patients for future genome-wide association studies (GWAS). The case definitions consisted of a combination of diagnoses, medications, and smoking history. ${ }^{25}$ By applying stringent criteria, the study results show a PPV of $95 \%$ and an NPV of $96 \%$ for identification of asthma cases and controls, using clinician review as the reference standard. GWAS require a high specificity, PPV, and NPV. A high specificity was achieved but at the loss of $24 \%$ of the potential asthma cases.

Vollmer et al used the electronic databases of a large health maintenance organization to develop a case definition for defining prevalent asthma and to validate it against chart review. ${ }^{26}$ The data systems of this organization, the Kaiser Permanente Northwest Division consist of both EHR (inpatient data, emergency department data, EpicCare) and administrative data: "Outside claims database" and "The outpatient pharmacy system". Table 2 presents the PPV of the eight different case definition algorithms to define asthma. The fourth case definition, based on a combination of an urgent care visit and the order of nebulizer treatment $(\mathrm{N}=25)$, had the highest PPV (100\%), while the first case definition, based on non-urgent care visits, $(\mathrm{N}=4,460)$ had a PPV of $95 \%$ while identifying a much larger population.
Donahue et al sought to determine the reliability of identifying asthmatics through automated medical and pharmacy records. All adult members of the Harvard Pilgrim Health Care program who received an asthma diagnosis and at least one asthma drug between 1988 and 1991 were identified. ${ }^{27}$ The authors manually reviewed records of a random sample of 100 patients to validate the asthma diagnosis. The PPV of a coded asthma diagnosis was $86 \%$.

Premaratne et al measured the validity of the string "asth" in the accident and emergency (A\&E) department attendance diagnosis field for identifying patients with asthma-related conditions attending the A\&E departments of two hospitals in the UK in $1995 .{ }^{28} \mathrm{~A}$ reception clerk entered the diagnosis field in a database at arrival in the A\&E department. The reference standard was a confirmation of the asthma diagnosis by a clinical officer, or symptoms of asthma plus a history of asthma or bronchodilators given with improvement, or a previously diagnosed asthmatic with symptoms or prescribed asthma medication. An "attendance diagnosis" of asthma was excluded if there was a clear alternative diagnosis or sufficient other evidence to exclude asthma. The string "asth" in the attendance diagnosis field had a sensitivity of $80 \%(75 \%-86 \%)$ and a specificity of $97 \%(96 \%-98 \%)$ for a confirmation of asthma.

\section{Linked databases}

Our search found two studies which used a second independent database to validate asthma diagnoses in the first database. The PPVs ranged from $46 \%$ to $58 \%$.

Coulter et a $\mathrm{l}^{30}$ compared repeat prescriptions for asthma, epilepsy, and thyroid disease with chronic disease registers stored on general practice computers in the early days of EHRs (1989). PPV of an asthma diagnosis on the register was $58 \%$ for asthma when using medication prescriptions as the reference standard.

Engeland et al evaluated the reliability of maternal disease registration (diabetes, asthma, and epilepsy) in the Medical Birth Registry of Norway (MBRN) ${ }^{29}$ The data they examined consisted of the EHRs of 108,489 pregnancies between April 2004 and January 2007. The reference standard was the prescriptions in the Norwegian Prescription Database (NorPD). The overall sensitivity of an asthma diagnosis in MBRN was 51\% (49-52), but increasing when considering with a higher asthma treatment step in the NorPD. The sensitivity was $40 \%$ when considering records which only used inhaled selective beta-2-adrenoreceptor agonists (step1), while the sensitivity of asthma diagnosis 
in records with systemic drugs other than adrenergics for obstructive airway diseases was $73 \%$.

\section{Questionnaires}

There was only one study which used a questionnaire as the reference standard for database validation.

Ward et al aimed to determine the degree of under- or over-reporting of the diagnosis of asthma for patients aged 16-55 years in one large general practice in the UK. ${ }^{31}$ The case definition described in Table 1, (based on either codes, text strings or prescriptions) yielded 833 potential asthma cases and 831 age- and sex-matched controls from the GP database. A questionnaire validated for the detection of bronchial hyper-reactivity was sent to all asthma patients and their matched controls. Patients with a diagnosis of asthma and bronchial hyper-reactivity in the questionnaire were considered to have asthma. Evidence of asthma was sought for two groups: patients with asthma and without symptoms of bronchial hyper-reactivity, and controls with symptoms of bronchial hyper-reactivity. The results show an overall PPV of the case definition of $89 \%$.

\section{Discussion}

The main finding of this review is that case definitions and methods of asthma diagnosis validation vary widely across different EHR databases. This is evident in the diversity of databases used by the studies, such as primary care databases, combined EHR and administrative databases, or data from nested case-control studies within larger cohorts. Some databases originate from a single or a few health centers, while others span millions of patients. The source of the EHR databases (primary care, secondary care, and urgent care) influences the case definition of asthma and the way the validation is conducted. Patients seeking care for asthma symptoms will present differently in each setting, and the test measures might reflect this.

Case definitions are designed with different purposes in mind, and each of the studied test measures (sensitivity, specificity, PPV, and NPV) have different uses. A high sensitivity is needed to identify all asthma patients from a database, but if the aim is to exclude all records of patients who do not have asthma, a high specificity is more important. ${ }^{32}$ The PPV is the proportion of true positives among all positive results: the patients who have asthma in the examined database who also have asthma according to the reference standard. The NPV shows the proportion of true negatives among all negative results: patients that do not have asthma in the database who also do not have asthma according the reference standard.
PPVs and NPVs are directly related to the prevalence of asthma in the population. The PPV will increase with rising prevalence; the NPV will decrease with rising prevalence assuming all other factors remain constant.

Studies, the main aim of which was not database validation, were able to demonstrate a high test measure to suit their specific needs (PPV, NPV, sensitivity or specificity greater than $80 \%$ ). If this was not the case, their main study results (not including validation) would not be reliable, and thus potential studies with low validity of asthma diagnosis might not have been conducted or published. In contrast, studies with a main aim of validation of asthma in databases have a wider range of test measures depending on the case definition. The PPV in these studies range from $46 \%{ }^{29}$ to $96 \% .{ }^{23}$

Manual validation was the most common reference standard in the validation studies included in this systematic review. The computer-generated case definitions studied recently by Engelkes et al, ${ }^{20} \mathrm{Afzal}$ et al, ${ }^{21}$ Dexheimer et al, ${ }^{22}$ and $\mathrm{Wu}$ et $\mathrm{al}^{23}$ provide ways to create algorithms with high sensitivities and specificities. The PPVs of these methods (whether a person identified as having an asthma diagnosis actually has asthma) might not be sufficient for all purposes $(63 \%-82 \%)$. Preselected case definitions were used in five out of ten studies which manually validated the databases. The studies by Xi et al, ${ }^{19}$ Kozyrskyj et al, ${ }^{24}$ Pacheco et al, ${ }^{25}$ Vollmer et al, ${ }^{26}$ Donahue et al, ${ }^{27}$ and Premaratne et $\mathrm{al}^{28}$ used this approach and all report at least one case definition algorithm with a PPV above $85 \%$. The best results arise when combining diagnostic data and prescription data.

Other studies by Engeland et $\mathrm{al}^{29}$ and Coulter et $\mathrm{al}{ }^{30}$ used an external data source as reference standard. This approach needs two databases with near complete data, so their test measures are reliable on the quality and completeness of the two databases. It also requires that the validity of the reference standard is already known. However, they are much cheaper to carry out overall. Manual validation requires a considerable amount of time to complete, and questionnaires to hundreds of patients or clinicians can be expensive or unreliable. Coulter et al measured database completeness and integrity by studying different diseases including asthma. Their focus was not on asthma validation, but rather to check whether a digital database can be a valid alternative for analog registration.

Typical problems of validation studies are the lack of availability of a reliable reference standard and the interdependence of different data sources used for validation. There were four studies, not included in this review, which used face validity to compare the prevalence of asthma 
using a case definition to the general asthma prevalence. This method was not considered sufficiently exact for inclusion $^{33-36}$ and by definition was unable to verify the validity of individual records.

The diagnosis of asthma can represent different conditions in different regions of the world. Thus, several authors used an inclusive strategy and many diagnosis codes in order to maximize sensitivity. Researchers must weigh the benefits of a case-finding algorithm with high sensitivity against the likely lower specificity and PPV, according to the purpose of their research. In future studies using predetermined case definitions, it may be of interest to evaluate the predictive value of a specific set of codes validated by chest physicians or GPs working in the health system the database originates from. This group may be more accurate when assigning the diagnosis, and the codes applied may yield a much higher predictive value than when evaluating the same group of codes assigned by all providers. The PPV, NPV, sensitivity, and specificity can differ greatly within a single study, as shown in the studies by Xi et al, ${ }^{19}$ Afzal et al, ${ }^{21}$ Kozyrskyj et al, ${ }^{24}$ and Vollmer et al. ${ }^{26}$ For this reason, the testing of multiple case definitions to obtain the algorithm with the highest test measure needed would be beneficial for future studies.

\section{Conclusion}

Asthma validation studies using EHRs are very varied in their approach to the validation. This seems driven by the nature of the data and the reference standards used. Machine-learning methods of algorithm development allow for measuring all elements of validity. Different case definitions within a single data source have different validity, highlighting the importance of testing a range of case definitions.

\section{Strengths and limitations of this study}

The review of validation of asthma diagnosis codes in EHRs informs selection of asthma definitions used by future studies and identify any gaps in quality and scope of validation studies. It also provides an overview of the case definitions and algorithms with their PPV, NPV, sensitivity or specificity.

Validated case definition algorithms are often very specific to the database they were developed in, limiting their generalizability.

Publication bias might be an issue as methods that do not find favorable results may be less likely to have been published.

\section{Data sharing statement}

Study data are available on request to FN.

\section{Acknowledgments}

This work was supported by GlaxoSmithKline (GSK), through a $\mathrm{PhD}$ scholarship for $\mathrm{FN}$ with grant number EPNCZF5310. The publishing of this study was supported by the Wellcome Trust: grant number 098504/Z/12/Z.

\section{Author contributions}

All authors made substantial contributions to conception and design, acquisition of data, or analysis and interpretation of data; took part in drafting the article or revising it critically for important intellectual content; gave final approval of the version to be published; and agree to be accountable for all aspects of the work.

\section{Disclosure}

FN and SW are funded by a GSK scholarship during their $\mathrm{PhD}$ programs. JKQ reports grants from MRC, BLF, Wellcome Trust, and has received research funds from GSK, AZ, Quintiles IMS and had personal fees from AZ, Chiesi, BI. HM is an employee of GSK R\&D and owns shares of GSK Plc. IJD is funded by, holds stock in, and has consulted for GSK. The authors report no other conflicts of interest in this work.

\section{References}

1. World Health Organization. Asthma Fact sheet No 307. Geneva: World Health Organization; 2013.Available from: http://www.who.int/ mediacentre/factsheets/fs307/en/.

2. Global initiative for asthma. [webpage on the Internet]. 2017 GINA Report, Global Strategy for Asthma Management and Prevention. Available from: http://ginasthma.org/2017-gina-report-global-strategyfor-asthma-management-and-prevention/.

3. Rabe KF, Adachi M, Lai CK, Soriano JB, Vermeire PA, Weiss KB, Weiss ST. Worldwide severity and control of asthma in children and adults: the global asthma insights and reality surveys. J Allergy Clin Immunol. 2004;114(1):40-47.

4. Demoly P, Gueron B, Annunziata K, Adamek L, Walters RD. Update on asthma control in five European countries: results of a 2008 survey. Eur Respir Rev. 2010;19(116):150-157.

5. Langan SM, Benchimol EI, Guttmann A, et al. Setting the RECORD straight: developing a guideline for the REporting of studies Conducted using Observational Routinely collected Data. Clin Epidemiol. 2013;5:29-31.

6. Denney MJ, Long DM, Armistead MG, Anderson JL, Conway BN. Validating the extract, transform, load process used to populate a large clinical research database. Int J Med Inform. 2016;94:271-274.

7. Rimland JM, Abraha I, Luchetta ML, et al. Validation of chronic obstructive pulmonary disease (COPD) diagnoses in healthcare databases: a systematic review protocol. BMJ Open. 2016;6(6):e011777.

8. Abraha I, Giovannini G, Serraino D, Fusco M, Montedori A. Validity of breast, lung and colorectal cancer diagnoses in administrative databases: a systematic review protocol. BMJ Open. 2016;6(3):e010409. 
9. Lo Re V 3rd, Haynes K, Forde KA, Localio AR, Schinnar R, Lewis JD. Validity of The Health Improvement Network (THIN) for epidemiologic studies of hepatitis C virus infection. Pharmacoepidemiol Drug Saf. 2009;18(9):807-814.

10. Weiskopf NG, Weng C. Methods and dimensions of electronic health record data quality assessment: enabling reuse for clinical research. J Am Med Inform Assoc. 2013;20(1):144-151.

11. Sharifi M, Krishanswami A, McPheeters ML. A systematic review of validated methods to capture acute bronchospasm using administrative or claims data. Vaccine. 2013;31 (Suppl 10):K12-K20.

12. Mullooly JP, Schuler R, Mesa J, Drew L, DeStefano F; VSD team. Wheezing lower respiratory disease and vaccination of premature infants. Vaccine. 2011;29(44):7611-7617.

13. Sanders DL, Gregg W, Aronsky D. Identifying asthma exacerbations in a pediatric emergency department: a feasibility study. Int J Med Inform. 2007;76(7):557-564.

14. Al Sallakh MA, Vasileiou E, Rodgers SE, Lyons RA, Sheikh A, Davies GA. Defining asthma and assessing asthma outcomes using electronic health record data: a systematic scoping review. Eur Respir J. 2017;49(6). pii: 1700204.

15. Nissen F, Quint JK, Wilkinson S, Mullerova H, Smeeth L, Douglas IJ. Validation of asthma recording in electronic health records: protocol for a systematic review. BMJ Open. 2017;7(5):e014694.

16. Whiting PF, Rutjes AW, Westwood ME, et al. QUADAS-2: a revised tool for the quality assessment of diagnostic accuracy studies. Ann Intern Med. 2011;155(8):529-536.

17. Tang PC, Ralston M, Arrigotti MF, Qureshi L, Graham J. Comparison of methodologies for calculating quality measures based on administrative data versus clinical data from an electronic health record system implications for performance measures. J Am Med Inform Assoc. 2007;14(1):10-15.

18. Angier H, Gold R, Gallia C, et al. Variation in outcomes of quality measurement by data source. Pediatrics. 2014;133(6):e1676-e1682.

19. Xi N, Wallace R, Agarwal G, Chan D, Gershon A, Gupta S. Identifying patients with asthma in primary care electronic medical record systems Chart analysis-based electronic algorithm validation study. Can Fam Physician. 2015;61(10):e474-e483.

20. Engelkes M, Afzal Z, Janssens H, et al. Automated identification of asthma patients within an electronical medical record database using machine learning. Eur Respir J. 2012;40:4655.

21. Afzal Z, Engelkes M, Verhamme KM, Janssens HM, Sturkenboom MC, Kors JA, Schuemie MJ. Automatic generation of case-detection algorithms to identify children with asthma from large electronic health record databases. Pharmacoepidemiol Drug Saf. 2013;22(8):826-833.

22. Dexheimer JW, Abramo TJ, Arnold DH, et al. Implementation and evaluation of an integrated computerized asthma management system in a pediatric emergency department: a randomized clinical trial. Int J Med Inform. 2014;83(11):805-813.
23. Wu ST, Sohn S, Ravikumar KE, Wagholikar K, Jonnalagadda SR, Liu $\mathrm{H}$, Juhn YJ. Automated chart review for asthma cohort identification using natural language processing: an exploratory study. Ann Allergy Asthma Immunol. 2013;111(5):364-369.

24. Kozyrskyj AL, HayGlass KT, Sandford AJ, Paré PD, Chan-Yeung M, Becker AB. A novel study design to investigate the early-life origins of asthma in children (SAGE study). Allergy. 2009;64(8):1185-1193.

25. Pacheco JA, Avila PC, Thompson JA, et al. A highly specific algorithm for identifying asthma cases and controls for genome-wide association studies. AMIA Annu Symp Proc. 2009;2009:497-501.

26. Vollmer WM, O'Connor EA, Heumann M, Frazier EA, Breen V, Villnave J, Buist AS. Searching multiple clinical information systems for longer time periods found more prevalent cases of asthma. J Clin Epidemiol. 2004;57(4):392-397.

27. Donahue JG, Weiss ST, Goetsch MA, Livingston JM, Greineder DK, Platt R. Assessment of asthma using automated and full-text medical records. J Asthma. 1997;34(4):273-281.

28. Premaratne UN, Marks GB, Austin EJ, Burney PG. A reliable method to retrieve accident and emergency data stored on a free-text basis. Respir Med. 1997;91(2):61-66.

29. Engeland A, Bjørge T, Daltveit AK, Vollset SE, Furu K. Validation of disease registration in pregnant women in the Medical Birth Registry of Norway. Acta Obstet Gynecol Scand. 2009;88(10):1083-1089.

30. Coulter A, Brown S, Daniels A. Computer held chronic disease registers in general practice: a validation study. J Epidemiol Community Health. 1989;43(1):25-28.

31. Ward DG, Halpin DM, Seamark DA. How accurate is diagnosis of asthma in a general practice database? A review of patients' notes and questionnaire-reported symptoms. Br J Gen Pract. 2004;54(507): 753-758.

32. Parikh R, Mathai A, Parikh S, Chandra Sekhar G, Thomas R. Understanding and using sensitivity, specificity and predictive values. Indian J Ophthalmol. 2008;56(1):45-50.

33. Hansell A, Hollowell J, McNiece R, Nichols T, Strachan D. Validity and interpretation of mortality, health service and survey data on COPD and asthma in England. Eur Respir J. 2003;21(2):279-286.

34. Leone FT, Grana JR, McDermott P, MacPherson S, Hanchak NA, Fish JE. Pharmaceutically-based severity stratification of an asthmatic population. Respir Med. 1999;93(11):788-793.

35. Pearson N, O’Brien J, Thomas H, Ewings P, Gallier L, Bussey A. Collecting morbidity data in general practice: the Somerset morbidity project. BMJ. 1996;312(7045):1517-1520.

36. Moth G, Vedsted P, Schiotz P. Identification of asthmatic children using prescription data and diagnosis. Eur J Clin Pharmacol. 2007;63(6):605-611.

37. Moher D, Liberati A, Tetzlaff J, Altman DG, PRISMA Group. Preferred reporting items for systematic Reviews and meta-analyses: the PRISMA statement. BMJ. 2009;339:b2535. 


\section{Supplementary material}

Algorithm used for literature review

Asthma validation in electronic health records: a systematic review

\section{MEDLINE}

1 (validat* or verif*).mp. [mp=title, abstract, original title, name of substance word, subject heading word, keyword heading word, protocol supplementary concept word, rare disease supplementary concept word, unique identifier]

2 (PPV or PNV or NPV or "positive predictive value*" or "negative predictive value*" or "predictive positive value*" or "predictive negative value*" or "likelihood ratio" or precision or accuracy or "receiver operating characteristic*" or ROC or kappa).mp. [mp=title, abstract, original title, name of substance word, subject heading word, keyword heading word, protocol supplementary concept word, rare disease supplementary concept word, unique identifier]

3 Validation Studies/ or validation.mp. or validation studies as topic/

4 (electronic* or digital* or computeri?ed or programmed or automated or database or data base).mp. $[\mathrm{mp}=$ title, abstract, original title, name of substance word, subject heading word, keyword heading word, protocol supplementary concept word, rare disease supplementary concept word, unique identifier]

5 asthma.mp. or Asthma/ or Asthma, Occupational/ or Asthma, Exercise-Induced/

6 Database Management Systems/

71 or 2 or 3

84 or 6

95 and 7 and 8

\section{EMBASE}

1 (validat* or verif*).mp. [mp=title, abstract, heading word, drug trade name, original title, device manufacturer, drug manufacturer, device trade name, keyword]

2 validation.mp. or validation study/ or validation process/

3 (sensitivity or specificity or "Sensitivity and Specificity"). $\mathrm{mp} .[\mathrm{mp}=$ title, abstract, heading word, drug trade name, original title, device manufacturer, drug manufacturer, device trade name, keyword]

4 (PPV or PNV or NPV or "positive predictive value" or "predictive negative value" or "negative predictive value" or "likelihood ratio" or precision or accuracy or "receiver operating characteristic" or ROC or kappa).mp. [mp=title, abstract, heading word, drug trade name, original title, device manufacturer, drug manufacturer, device trade name, keyword, floating subheading]

5 (electronic* or digital* or computeri?ed or programmed or automated or database or data base).mp. [mp=title, abstract, heading word, drug trade name, original title, device manufacturer, drug manufacturer, device trade name, keyword]

6 mild persistent asthma/ or nocturnal asthma/ or experimental asthma/ or moderate persistent asthma/ or severe persistent asthma/ or Asthma.mp. or exercise induced asthma/ or occupational asthma/ or intrinsic asthma/ or asthma/ or allergic asthma/ or extrinsic asthma/ or mild intermittent asthma/

71 or 2 or 3 or 4

85 and 6 and 7
Clinical Epidemiology

\section{Publish your work in this journal}

Clinical Epidemiology is an international, peer-reviewed, open access, online journal focusing on disease and drug epidemiology, identification of risk factors and screening procedures to develop optimal preventative initiatives and programs. Specific topics include: diagnosis, prognosis, treatment, screening, prevention, risk factor modification,

\section{Dovepress}

systematic reviews, risk and safety of medical interventions, epidemiology and biostatistical methods, and evaluation of guidelines, translational medicine, health policies and economic evaluations. The manuscript management system is completely online and includes a very quick and fair peer-review system, which is all easy to use. 